\title{
Evaluación de la competencia de trabajo en equipo en los grados de ingeniería
}

\section{Assessment of teamwork skills in engineering degree course}

\author{
Elisabeth Viles, Carmen Jaca, Joseba Campos, Nicolás Serrano y Javier Santos \\ Dpto.de Organización Industrial.TECNUN. Escuela de Ingenieros. Universidad de Navarra. \\ Po de Manuel Lardizábal. San Sebastián. \\ eviles@tecnun.es, cjaca@tecnun.es, jcampos@tecnun.es, nserrano@tecnun.es, jsantos@tecnun.es
}

Fecha de recepción: 6-10-2010

Fecha de aceptación: 7-3-2011

Resumen: Este artículo presenta un modelo adaptado de la bibliografía para medir la aplicación de los factores que promueven la efectividad del trabajo en equipo. Se quiere dar a conocer la experiencia real del desarrollo y evaluación de trabajos en equipo realizados con alumnos de ler curso de distintos grados de Ingeniería durante el curso académico 2009- I0. La planificación de los trabajos en equipo se desarrolló teniendo como premisa cómo serían evaluados. Los resultados de la evaluación han sido recogidos de manera cualitativa y cuantitativa. El artículo presenta además, las conclusiones más interesantes de esta experiencia.

Palabras clave: efectividad del trabajo en equipo, competencias, evaluación,educación superior.

\begin{abstract}
This article presents a model, adapted from the literature, to measure the application of factors that foment teamwork effectiveness. The goal of this article is to publish the actual experience of developing and assessing teamwork activities which were carried out during the past academic year, 2009- I0, by freshmen students at an Engineering School. The teamwork planning was developed taking into account how it would be evaluated. The assessment results have been quantitatively and qualitatively analyzed. Finally, the paper shows the most relevant conclusions.
\end{abstract}

Key Words: team working effectiveness, abilities, evaluation, High School \& University.

\section{Introducción}

El trabajo en equipo está considerado como una de las herramientas más poderosas para la consecución de objetivos en cualquier ámbito, sector o actividad. En las organizaciones, el trabajo en equipo supera al trabajo individual cuando la tarea a realizar es compleja y se necesita del conocimiento, habilidades, creatividad y compromiso del conjunto para alcanzar el objetivo (Salas et al., 2005). Dentro de las competencias a desarrollar por los ingenieros en los nuevos planes de estudio están las relacionadas con el liderazgo y el trabajo en equipo (Maffioli y Augusti, 2003; Marin-Garcia et al., 2009; Wanous et al., 2009). Más allá de inculcar el trabajo y desarrollo individual, la universidad como institución, debería servir como plataforma para la formación y entrenamiento de los futuros profesionales en esta forma de trabajar, a través de sus planes de estudio y guías docentes.

Se podría analizar cuantitativamente en qué porcentaje de asignaturas de los planes docentes existentes por universidad se plantean algún trabajo en equipo, como forma de conseguir los objetivos planteados en dichas asignaturas, tanto formativos como de aptitudes. Sin embargo, aunque resulta evidente que la escuela y la universidad fomentan el desarrollo de trabajo en equipo, pocas veces se cuestiona que el proceso de trabajar en equipo también debiera ser medido y evaluado. Es decir, se utiliza la herramienta pero no se llega a evaluar la enseñanza y el aprendizaje de la misma, ni mucho menos la efectividad del propio proceso de trabajo en equipo.

En este contexto es necesario formar a los alumnos de ingeniería en habilidades de trabajo en equipo, a la vez que se evalúa la efectividad del equipo tanto en términos de resultados como de las propias habilidades ligadas al trabajo en equipo.

Este artículo presenta la experiencia desarrollada en la Escuela de Ingenieros de la Universidad de Navarra, dentro del primer curso común a nueve grados. Para el desarrollo y evaluación de la competencia de trabajo en equipo se han formado equipos de alumnos para realizar trabajos para dos asignaturas dis- 
tintas, que luego se han evaluado en función de varios criterios asociados con la efectividad del trabajo en equipo.

\section{Modelo integrado de la efectividad del trabajo en equipo}

Como punto de partida en este estudio ha sido necesario consensuar el concepto y la definición de trabajo en equipo. En la bibliografía analizada se pueden encontrar diferentesdefiniciones del trabajo en equipo. La mayoría de ellas resaltan algunas de las características que debe contener el trabajo en equipo tales como: existencia de objetivos comunes, esfuerzo conjunto para alcanzar los objetivos, ambiente donde se fomente la comunicación y el intercambio de ideas, toma de decisiones en conjunto, etc (Hackman, 1990; Salas et al., 2005; Xyrichis y Ream, 2008; Zander, 1994). Ladefinición de equipo utilizada en este estudio ha sido la siguiente:

Se llama equipo de trabajo a un pequeño grupo de personas con habilidades y conocimientos complementarios, que están comprometidos con una misión, un conjunto de metas de desempeño y con un enfoque común, para los cuales son mutuamente responsables (Katzenbach y Smith, 1993). El trabajo en equipo implica tanto los resultados individuales como los que se producen de manera colectiva. Por tanto, los grupos se convierten en equipos cuando desarrollan un sentido compartido y utilizan la sinergia entre sus miembros.

Por tanto, con el objetivo principal de desarrollar la competencia de trabajo en equipo en los grados de ingeniería, se han formado equipos de alumnos a los que se han asignado varios proyectos. Se evaluarán tanto el resultado del trabajo de estos equipos como su eficiencia en el propio trabajo en equipo.

Para la composición y establecimiento de los equipos de trabajo de alumnos se ha utilizado un modelo adaptado de la bibliografía para medir la efectividad del trabajo en equipo (Mathieu et al., 2008). Se trata de un modelo de desarrollo retroalimentado que ilustra la forma en la que los equipos cambian y son influenciados por varios factores al interactuar en el tiempo. Este modelo se representa en la Figura I.

En este modelo se agrupan los factores que afectan al equipo en tres grandes grupos: inputs, mediators y outcomes (Modelo IMO).

En los inputs se agrupan los factores previos a la formación del equipo. Estos factores pueden clasificarse en tres niveles: miembros, equipo o contexto organizativo. En el modelo se observa que los tres grupos están anidados. De esta forma se representa la fuerte influencia que tienen los factores del con-

Figura I

Modelo IMO (Input- Mediators-Outcome) del desarrollo del trabajo en equipo

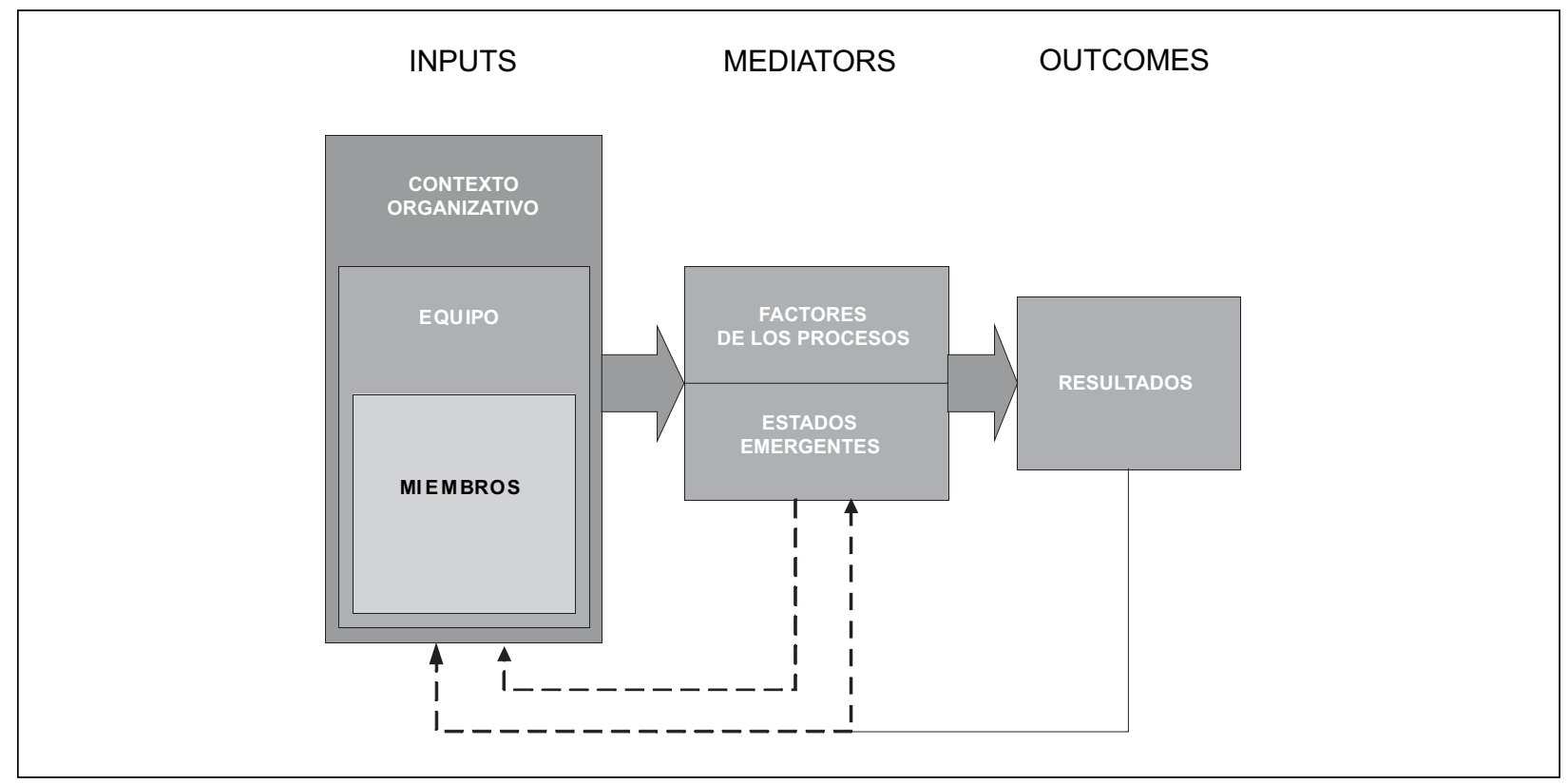


texto organizativo sobre el equipo y, a su vez, el equipo influye sobre los miembros exigiéndoles ciertas competencias y distribución de roles. El contexto organizativo hace referencia a todos aquellos factores que existen dentro de la organización y que influyen en el diseño de los equipos y en el entorno de trabajo. Los factores pertenecientes al equipo son aquellos que están relacionados con el funcionamiento del propio equipo, que están definidos o existen antes de que el equipo empiece a trabajar como tal. Por otro lado se incluyen las características individuales de los miembros del equipo en los factores pertenecientes a los miembros.

En los mediators se incluyen los factores que influyen durante el proceso del trabajo en equipo. Algunos de estos factores suponen acciones de los miembros mientras que otros se consideran como estados afectivos, cognitivos o referentes a la motivación. Es por ello que los mediators se dividen en factores de procesos y en estados emergentes.
Por último, en los outcomes se agrupan los factores relacionados con el desempeño del trabajo en equipo y, por tanto, se valoran los resultados tanto del equipo como de los miembros y de la organización.

En el modelo se representa mediante bucles de retroalimentación la característica cíclica de los procesos realizados por los equipos. Las líneas sólidas de retroalimentación sugieren que los resultados influyen de manera importante en los procesos y estados emergentes. Las líneas punteadas indican que la influencia sobre los inputs es menor, al ser más difícil de cambiar la estructura y composición del equipo así como el contexto organizativo.

Para poder medir la eficacia del trabajo en equipo ha sido necesario identificar factores que puedan ser valorados en cada uno de los grupos contenidos dentro de los inputs, mediators y outcomes del modelo. En la Figura 2 se listan todos los factores que se han identificado en el modelo organizados de acuerdo a

Figura 2

Modelo IMO para medir la efectividad del trabajo en equipo con los factores identificados

\begin{tabular}{|c|c|c|}
\hline INPUTS & MEDIAT ORS & OUTCOMES \\
\hline  & \begin{tabular}{l}
\multicolumn{1}{c}{$\begin{array}{l}\text { FACTORES } \\
\text { DE PROCESOS }\end{array}$} \\
- Participación \\
- Gestión de conflictos \\
- Toma de deciciones \\
- Comunicación externa \\
- Comunicación interna \\
- Colaboración, cooperación \\
- Coordinación \\
- Resolución de problemas \\
- Liderazgo \\
\multicolumn{1}{c}{ ESATADOS } \\
EMERGENTES \\
- Aprendizaje del equipo \\
- Cohesión \\
- Ambiente del equipo \\
- Respeto mutuo \\
- Confianza \\
- Motivación \\
- Modelos mentales comunes
\end{tabular} & $\begin{array}{l}\text { RESULTADOS } \\
\text { - Resultados de los miembros } \\
\text { - Retroalimentación de los } \\
\text { miembros } \\
\text { - Resultados del equipo } \\
\text { - Retroalimentación del equipo } \\
\text { - Resultados de la organización } \\
\text { - Retroalimentación de la } \\
\text { organización }\end{array}$ \\
\hline
\end{tabular}


los inputs, mediators y outcomes. Actualmente existen pocos estudios donde se analicen modelos IMO y se identifiquen los factores que caracterizan el buen desempeño de los equipos de trabajo. Para la identificación y definición de los factores que se presentan en este trabajo se ha combinado el conocimiento de los autores con el estudio de distintas fuentes bibliográficas (Cohen y Bailey, 1997; Lemieux-Charles y McGuire, 2006; Mathieu et al., 2008)

\section{Fases de preparación del proyecto}

Este artículo detalla cómo se ha desarrollado la planificación de los trabajos en equipo encomendados a los alumnos de ler curso de distintos grados de Ingeniería, para después aportar los resultados de la evaluación que han sido recogidos de manera cualitativa y cuantitativa.

De acuerdo al modelo anterior, los factores asociados a las entradas o inputs del modelo son parte importante del modelo de efectividad de los equipos. Estos factores se refieren al estado inicial de preparación de la organización, equipos y personas para conseguir dar soporte y apoyo al desarrollo de la actividad de grupo. Luego, como puede verse en la Figura 2, estos factores se agrupan a su vez en tres grupos diferenciados: los factores asociados al contexto organizativo, los factores asociados al equipo y por último, los asociados a los que formarán parte del mismo.

En el caso que aquí se presenta, el contexto organizativo parte de los profesores involucrados en las asignaturas relacionadas con los trabajos a realizar, que en este caso pertenecen al mismo Departamento. Estos asumieron su tarea de cumplir con aquellos factores que desde la literatura aparecen como favorecedores para el buen desarrollo de la actividad del trabajo en grupo. Luego, se procuró en todo momento que los alumnos sintieran el apoyo del entorno organizativo: los profesores apoyaron concienzudamente la realización del trabajo, proporcionando a los alumnos los objetivos, las reglas y las estrategias que se seguirían para la realización de los mismos. Al mismo tiempo se detalló cómo serían evaluados y «recompensados» con estos trabajos.

Otro punto previo importante es la formación. Diversos estudios corroboran la idea de que la formación dirigida a trabajar en equipo influye positivamente en la efectividad del trabajo. La organización se aseguró que los alumnos recibirían una formación inicial sobre Trabajo en Equipo. Esta formación se impartió en una tercera asignatura también común a todos los grados y consistió en 6 horas de formación teórico-práctica, donde se puso el acento en la importancia del reparto de roles dentro de los equipos, con especial énfasis en la importancia del líder, y en la necesidad de establecer objetivos y estrategias comunes antes de comenzar cualquier trabajo en común.

Fue a través de esta formación donde se concienció también a los alumnos de la importancia que tiene en los equipos la planificación, el establecimiento de reglas internas de funcionamiento, la existencia de cierta autonomía en la toma de decisiones, etc., por lo que se instó a que cada grupo, previo al desarrollo del trabajo concreto de cada asignatura, a que firmara un contrato en el que cada equipo debía detallar qué roles asumiría cada componente del grupo. de qué actividades se encargaría, cuáles eran sus reglas de actuación, etc.

En cuanto a la composición de los grupos, y siguiendo también las directrices del modelo que relaciona la efectividad del equipo con determinadas pautas en la formación del mismo, como son: rasgos personales, la diversidad de los componentes y las distintas capacidades de las personas, se consideró oportuno crear grupos de trabajo con el siguiente criterio: diversidad de conocimientos (basado en notas previas de los alumnos), equilibrio en sexo y diversidad de origen geográfico. Finalmente se formaron 61 equipos de trabajo de 4 ó 5 alumnos cada uno. Se mantuvo la misma composición de equipos para ambas asignaturas con el objetivo de poder evaluar el aprendizaje del equipo a trabajar en equipo, al estar distanciadas en tiempo las dos prácticas, dándoles asimismo opciones a modificar su estrategia, planificación y reglas de una práctica a la otra.

De la misma manera, y tal y como aparece en la Figura 2, para medir y la valorar la eficiencia del trabajo en equipo, la evaluación, así como la «recompensa» que se deriva de los resultados obtenidos por los equipos, debe hacerse no sólo a nivel de equipo, sino también a nivel individual.

Esta manera de proceder provocó el diseño de una estrategia diferente a la habitual de valorar los trabajos realizados. Se quería valorar no sólo la realización del trabajo, sino también el desarrollo del mismo. Además, esta evaluación debería permitir 
diferenciar el trabajo aportado por cada miembro del equipo. Por tanto, se siguió la siguiente estrategia de evaluación:

- El trabajo para cada asignatura se valoraba conjuntamente, lo cual proporcionaba una nota inicial que los miembros de cada equipo debían repartirse entre ellos de acuerdo a las reglas que se habían establecido en el contrato inicial y en el desarrollo real del trabajo realizado.

- Además cada miembro del equipo tenía la oportunidad de valorar cualitativamente el trabajo del resto del grupo (evaluación intragrupo o $360^{\circ}$ ) en cuanto a su calidad, compromiso en la realización y actitud hacia el resto del grupo. Esta calificación personal e individual posteriormente ha permitido a los profesores ver la relación existente entre el reparto de la nota y la percepción real del grupo para con cada miembro del mismo.

Los criterios de evaluación al trabajo en grupo fueron claramente expuestos y entregados a los alumnos con anterioridad al desarrollo del mismo. Estos contenían los porcentajes asignados a cada parte.

Finalmente y al término del trabajo y su calificación, todos los alumnos participantes en esta experiencia fueron sometidos a una encuesta de satisfacción, en la que se trató de cuantificar cómo se habían desarrollado los factores mediators del modelo, tanto los factores relacionados con los procesos: participación, gestión de conflictos, comunicación, etc. como los estados emergentes respecto si ha habido aprendizaje, cohesión, respeto mutuo, etc.

\section{Resultados}

Los resultados obtenidos de esta experiencia son abundantes y de diversa tipología. Es decir, por un lado están los resultados numéricos como consecuencia de las calificaciones obtenidas por los alumnos en estos trabajos. También existen resultados derivados de la observación de la evolución de los equipos de trabajo (de un trabajo al siguiente) en cuanto a sus estrategias, planificación y modo de resolver sus conflictos. Por otro lado, se dispone de la información de los propios alumnos recogida a partir de un cuestionario anónimo, que intenta cuantificar la impresión del alumno respecto de esta novedosa forma de trabajar y ser evaluados. Finalmente, se dispone de la valoración cualitativa de los profesores involucrados en esta experiencia.

\section{I. Datos acerca del comportamiento de los equipos y su evolución en cuanto a estrategias y resolución de conflictos en el paso de un trabajo al siguiente}

Para comprender mejor el análisis que se describe a continuación conviene recordar los datos que los profesores encargados de los trabajos han podido disponer para su análisis y evaluación.

Por un lado, y como es lo habitual, se disponía de la calificación del profesor al grupo por su trabajo realizado. Tradicionalmente, en los trabajos académicos que son realizados en grupo, el reparto de la nota entre sus miembros es a partes iguales; es decir, todos los miembros del grupo reciben la misma calificación por el trabajo realizado. Sin embargo, en esta experiencia, los miembros del grupo podían ser calificados de manera diferente de acuerdo a su implicación, trabajo y responsabilidad con el trabajo y esta asignación debía establecerse dentro del propio grupo por consenso. Por tanto el profesor ha dispuesto finalmente de la calificación del grupo inicial y la calificación final de cada alumno, además de la evaluación cualitativa para cada miembro realizada por el resto de compañeros de su grupo. Asimismo disponía también de una copia del contrato firmado inicialmente por cada grupo, donde se establecían las reglas que regirían la realización del trabajo y el reparto final de las notas.

Toda esta información ha permitido analizar, tanto de forma cualitativa como cuantitativa, el comportamiento de los grupos y su evolución en estrategia de un trabajo al siguiente.Y como resultado, se dispone de una información basada en datos acerca del aprendizaje de los alumnos en esta competencia.

Tras esta experiencia los datos reflejan lo siguiente:

- La evidencia de la existencia de conflictos en los grupos, lo que da lugar a grupos no cohesionados. El conflicto asociado a este tipo de trabajos está relacionado con la poca implicación de alguno o varios de los miembros, que llegan a «aprovecharse/beneficiarse» (si reciben la misma calificación) del trabajo del resto.

- De acuerdo a la forma de evaluar descrita en esta experiencia, se pueden distinguir entre dos estados distintos del conflicto: conflictos latentes (aquellos en los que las valoraciones cualitativas intragrupo diferencian a unos de los otros, pero que no tiene efecto en el reparto de las calificaciones dentro del grupo) y conflictos públicos 
(aquellos que claramente penalizan o premian a alguno de sus miembros en la calificación cualitativa y en la cuantitativa).

- Tras la realización de dos trabajos distintos con cada grupo, los datos confirman la existencia de un tercio de grupos no cohesionados en cada trabajo (han tenido algún tipo de conflicto), que en el cómputo global para los equipos pasa a ser un $50 \%$.

- Concretamente, los datos acerca del primer trabajo realizado recogen un 33\% de grupos con conflicto (latente y público): el I $8 \%$ de los grupos reinciden en el siguiente trabajo, mientras que el $15 \%$ restante resuelve su conflicto. En el segundo trabajo aparecen un nuevo grupo de equipos con conflicto ( $13 \%$ grupos conflictivos de nueva aparición). La Figura 3 sintetiza la evolución de los grupos no cohesionados o conflictivos de un trabajo al otro.

- Finalmente es de reseñar que, teniendo en cuenta la calificación grupal, el 50\% de los grupos con conflictos públicos obtuvieron su calificación de grupo dentro del primer cuartil (el del 25\% de los grupos con calificaciones más bajas); el $70 \%$ por debajo de la media. La calificación final de cada miembro de esos grupos con el reparto, ha originado la permanencia en el primer cuartil de las personas «conflictivas», mientras el resto de componentes del grupo han pasado al cuarto cuartil (el del 25\% de alumnos con mejores calificaciones).

\subsection{Datos acerca de la satisfacción de los alumnos respecto de la experiencia}

Finalizados los trabajos y siendo públicas las calificaciones, se diseñó una encuesta de satisfacción para los alumnos participantes en esta experiencia. Cada pregunta debía responderse de acuerdo a una escala de I a 5 ( I- Nada de acuerdo; 5-Totalmente de acuerdo). La encuesta fue respondida por un $75 \%$ de la población analizada.

El objetivo principal de la encuesta era conocer de primera mano si veían necesaria la formación sobre trabajo en grupo (Figura 4), si el establecimiento de reglas iniciales para el grupo lo consideraban imprescindible para reducir el riesgo de conflictos posteriores (Figura 5) y si, en definitiva, valoraban en general la experiencia positivamente (Figura 6 y Figura 7). No obstante, se aprovechó la encuesta para obtener más datos acerca de la planificación de los trabajos (tiempos, recursos disponibles, material entregado,...) por parte de los profesores, los criterios de evaluación adoptados y el aprendizaje de los alumnos respecto de las asignaturas y de la propia competencia de trabajo en grupo.

Evolución de los grupos con conflicto de un trabajo al otro




Figura 4

«La formación recibida acerca de trabajar en grupo ha sido de mi interés»

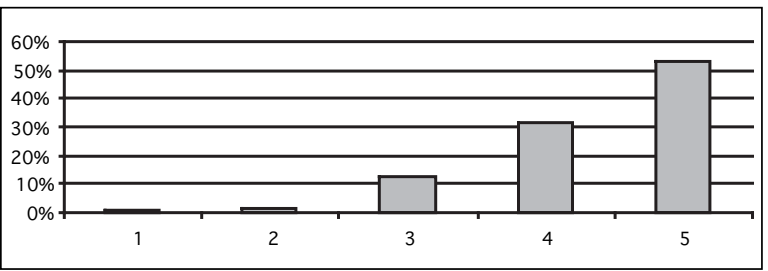

Figura 5

«Es imprescindible poner normas de trabajo, para que éste llegue a buen fin»

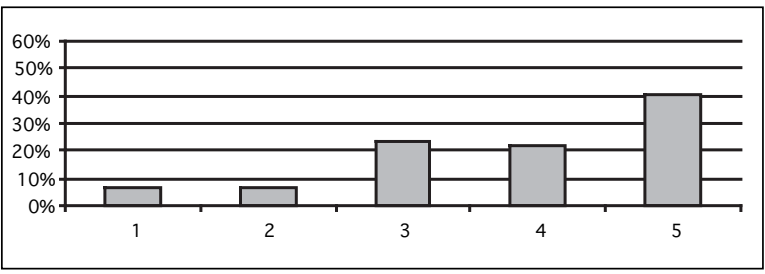

Figura 6

«El trabajo me ha hecho ser más consciente de lo que es trabajar en equipo»



Figura 7

«Teniendo en cuenta globalmente el tiempo de dedicación del trabajo, el aprendizaje adquirido por el mismo acerca de la asignatura, el aprendizaje de trabajar en grupo, considero interesante la realización de este tipo de trabajos en grupo»

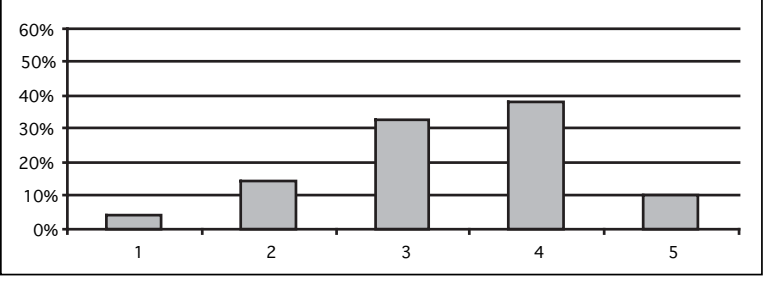

\subsection{Valoración cualitativa de los profesores}

Como resultado de esta experiencia se ha conseguido, no solo disponer de la calificación por traba- jo realizado, sino también evaluar la competencia de trabajo en grupo e incluso disponer de datos objetivos acerca de comportamientos sociales relacionados con nuestros jóvenes procedentes de un entorno muy concreto y definido.

Además de las impresiones y valoraciones ya realizadas, se podría reseñar lo siguiente:

- Acerca de los contratos. Los contratos previos que realizaban los alumnos para establecer sus normas de juego, han resultado ser muy creativos. En muchos se entraba al detalle de penalizaciones en nota por faltas de comportamiento tales como impuntualidades o falta de interés, que algunos han aplicado.

- Acerca de la formación. Se proporcionó una formación básica en trabajo en equipo, que ha sido bien valorada por los alumnos. Aun así y después de ver los resultados, conviene plantearse si debiera extenderse en tiempo y temario esta formación inicial. Hablar de resolución de conflictos o de cómo realizar reuniones de grupo efectivas son dos de los campos en los que se ha detectado carencias.

- Acerca de los trabajos. Una de los aspectos positivos desde el punto de vista más académico es haber conseguido una manera de valorar no sólo al grupo sino también valorar la participación de cada miembro dentro del grupo. Esto puede potenciar el uso de la herramienta a niveles académicos; el profesor tiene más certeza de que el alumno es merecedor de la nota conseguida, y en cierta manera se reducen o por lo menos se gestionan este tipo de conflictos donde algunos alumnos se benefician del trabajo de los demás.

- Acerca de los alumnos en general. Se valora positivamente la participación, motivación e implicación de los alumnos en la realización de los trabajos (sólo un 6\% de alumnos no ha trabajado al nivel del resto). Se ha conseguido unificar a la clase, provocando la necesidad de abrirse al resto de los compañeros y trabajar con gente «desconocida» (un 65\% ha valorado positivamente estos aspectos del trabajo). Por otro lado, se les ha dado autonomía (han podido premiarse y castigarse entre ellos, de acuerdo a sus normas. Un $80 \%$ de alumnos ha valorado positivamente la idea de poder repartirse la puntuación entre los miembros del grupo de acuerdo a su implicación en el trabajo). 


\section{Conclusiones}

Con el objetivo principal de desarrollar la competencia de trabajo en equipo en los grados de ingeniería de la Escuela de Ingenieros de la Universidad de Navarra, se han formado equipos con los alumnos de ler grado a los que se han asignado varios proyectos. Se han evaluado tanto el resultado del trabajo de estos equipos como su eficiencia en el propio trabajo en equipo.

Como aspectos positivos a concluir de la experiencia se apuntan los siguientes:

- Se ha constatado el hecho de que los alumnos cuando llegan a la universidad no saben trabajar en equipo, luego necesitan formación concreta sobre esta forma de trabajar.

- Los alumnos valoran positivamente que la evaluación de los trabajos permita la evaluación individual.

- La creación inicial de los grupos por parte de los profesores les ha permitido unirse más como clase, a la vez de aprender a trabajar con personas inicialmente «desconocidas».

- La realización de los trabajos con esta manera novedosa de proceder, les ha permitido ser más conscientes de lo que puede ser trabajar en grupo.

- A nivel académico, se ha conseguido homogenizar y nivelar al grupo en el conocimiento del temario asociado al trabajo realizado.

Sin embargo, siguen existiendo todavía puntos débiles que fortalecer. Se han detectado los siguientes:

- La formación acerca de cómo trabajar en grupo debiera contener sesiones específicas acerca de cómo conseguir tener reuniones de trabajo efectivas, temas de negociación y resolución de conflictos.

- A nivel académico, incluso modificando la forma de evaluar, los trabajos en grupo siguen penalizando en la nota global de la asignatura a los alumnos más brillantes. Es conveniente seguir buscando la fórmula de evaluación que, sola o en combinación con otras evaluaciones, reduzca o elimine estos desajustes.
Por otro lado, es deseable que los resultados de la evaluación de la efectividad del trabajo en equipo permitan mejorar la propuesta de trabajo en equipo de los nuevos grados. Se pretende, por tanto, que esta experiencia sirva de referencia para hacer una evaluación continuada que permita conocer el grado de desarrollo de la competencia de trabajo en equipo a lo largo de los siguientes cursos, de esa misma población de alumnos.

Al mismo tiempo, se espera que finalmente esta enseñanza repercuta sobre los propios alumnos y sobre las organizaciones en las que ellos desarrollen su vida profesional. Que esta manera de trabajar la asuman como propia y sean capaces de generar sinergias con un enfoque común en los grupos en los que les toque trabajar en su futuro.

\section{Bibliografía}

COHEN, S. G. y BAILEY, D. E. ( 1 997). «What Makes Teams Work: Group Effectiveness Research from the Shop Floor to the Executive Suite». Journal of Management, 23 (3) pp. 239-290.

HACKMAN, J. R. ( 1990). Groups that Work (and those that Don't) : Creating Conditions for Effective Teamwork. San Francisco: Ed.Jossey-Bass.

KATZENBACH, J. R. y SMITH, D. K. ( 1993). The Discipline of Teams. Harvard Business Review, 7| (2), I | I- I 20.

LEMIEUX-CHARLES, L. y MCGUIRE,W. L. (2006). «What do we Know about Health Care Team Effectiveness? A Review of the Literature». Med. Care Res. Rev., 63 (3) pp. 263-300.

MAFFIOLI, F. y AUGUSTI, G. (2003). «Tuning Engineering Education into the European Higher Education Orchestra». European Journal of Engineering Education, 28 (3) pp. 251.

MARIN-GARCIA, J.A., GARCIA-SABATER, J. P., PERELLOMARIN, M. R., CANOS-DAROS, L. (2009). «Propuesta De Competencias Para El Ingeniero De Organización En El Contexto De Los Nuevos Planes De Estudio». Intangible Capital, 5 (4) pp. 387-406.

MATHIEU, J., MAYNARD, M.T., RAPP,T., GILSON, L. (2008). «Team Effectiveness 1997-2007: A Review of Recent Advancements and a Glimpse into the Future». Journal of Management, 34 (3) pp. 4l0-476.

SALAS, E., SIMS, D. E., BURKE, C. S. (2005). «ls there a "Big Five" in Teamwork?». Small Group Research, 36 (5) pp. 555-599. 
WANOUS, M., PROCTER, B., MURSHID, K. (2009). «Assessment for Learning and Skills Development:The Case of Large Classes». European Journal of Engineering Education, 34 (I) pp. 77.
XYRICHIS, A. y REAM, E. (2008). «Teamwork: A Concept Analysis». J. Adv. Nurs., 6I (2) pp. 232-24I.

ZANDER, A. (1994). Making Groups Effective. San Francisco; Oxford: Ed.Jossey-Bass. 